\title{
Pesquisa-Aprendizagem no Ensino da Política e Gestão de Saúde: Relato de uma Experiência com e-Learning ${ }^{1}$
}

\author{
Search and Learning in theteaching of \\ Health Policies and Managing: An \\ Experience with e-Learning
}

Juan Stuardo Yazlle Rocha ${ }^{1}$ Maria do Carmo G. Caccia-Bava ${ }^{2}$ Carlos Eduardo M. de Rezende ${ }^{3}$

PALAVRAS-CHAVE

- Educação Médica;

- Educação à Distância;

- Medicina Social.

\section{KEY-WORDS}

- Education, Medical;

- Education, Distance;

- Social Medicine.

Recebido em: 18/04/2005

Reencaminhado em: 22/11/2005

Reencaminhado em: 15/12/2005

Aprovado em: 13/01/2006

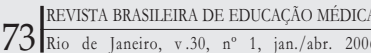

\footnotetext{
${ }^{1}$ Prêmio Excelência em Educação à Distância Abed \& Embratel, 2004 (3ํlugar - Relato de Experiência). Apresentado no $11^{\circ}$ Congresso Internacional de Educação à Distância - Salvador, Bahia, 7 a 10 de setembro de 2004.

${ }^{1}$ Médico, Assistente Social, Professor Titular, Departamento de Medicina Social, Faculdade de Medicina de Ribeirão Preto, Universidade de São Paulo, Ribeirão Preto, São Paulo, Brasil.

${ }^{2}$ Professora Doutora em Enfermagem em Saúde Pública, Departamento de Medicina Social, Faculdade de Medicina de Ribeirão Preto, Universidade de São Paulo, Ribeirão Preto, São Paulo, Brasil.

${ }^{3}$ Médico, Mestre em Ciências Médicas, Departamento de Medicina Social, Faculdade de Medicina de Ribeirão Preto, Universidade de São Paulo, Ribeirão Preto, São Paulo, Brasil.
}

\begin{abstract}
The authors present the results of an experience, transforming a traditional subject of medical education directed to health policies and management using information and communication technologies (e-Learning). Based on constructivist ideas about learning and education, the course pays special attention to the motivation of the student, to collaborative work, and to the search and elaboration of information from official sources. Characteristics of the course are: to motivate the students by proposing to characterize the health system of a city of their own choice; to promote studies and investigations in health using guidebooks directed to analysis and management of the health care system; indicate and show how to use official sites of health information and create a virtual research and learning environment available all the time. After four years of experience and for being well accepted among the students, this course model was expanded to other courses of the health area of FMRP-USP.
\end{abstract}




\section{INTRODUÇÃO}

Por várias razões, apontadas a seguir, é problemática a pesquisa e aprendizagem do sistema de saúde no Brasil no ensino de graduação em carreiras da área da saúde. A principal dificuldade é a inserção pontual dos serviços acadêmicos no sistema de saúde, o que dificulta a compreensão mais ampla da saúde no País - e a motivação do corpo discente. Também é difícil o acesso a dados, informações, documentos e resoluções na área da saúde se não houver disponibilidade de recursos de informática e comunicação.

Não é de estranhar, pois, que temas como a política de saúde, a conformação do Sistema Único de Saúde (SUS), as relações entre o público e o privado, os problemas do financiamento da assistência, os planos de saúde, os custos, a organização regional, a descentralização e outros - com os quais o futuro profissional irá conviver - pareçam assuntos "teóri$\cos ^{\prime \prime}$, distantes da realidade do aluno.

Focar o ensino no sistema de saúde do município onde o aluno mora (ou pretende trabalhar) e trazer aquela realidade de saúde à sala de aula, exercitar a elaboração de dados e informações para a compreensão do sistema são uma estratégia possível, tendo em vista a quantidade enorme de dados e informações, disponibilizados pela internet, provenientes dos níveis municipal, estadual e federal, como também internacional. Conhecer o sistema de saúde passa, então, pelas atividades de pesquisa e aprendizagem, com supervisão docente praticando a análise, síntese e avaliação dos elementos que caracterizam a organização e o funcionamento do sistema.

\section{ANTECEDENTES DA TRANSFORMAÇÃO DO CURSO}

Em 1975, o Departamento de Medicina Social (DMS) da Faculdade de Medicina de Ribeirão Preto da USP (FMRP-USP) criou a disciplina de Organização e Administração em Saúde, OAS - RCG 452, com o escopo de dar a conhecer as principais características do sistema de saúde no Brasil.

Oferecida no décimo semestre do curso de graduação em Medicina, possuía 72 horas de carga horária, distribuídas ao longo de nove semanas (dois períodos semanais de quatro horas, duas turmas por ano), o que permitia implementar alguns trabalhos práticos: levantar a expectativa dos alunos em relação ao mercado de trabalho, analisar o processo de assistência ambulatorial, discutir os sistemas de saúde em outros países, e modelos de previsão de ocorrências e custos. Entretanto, a baixa vivência dos alunos acerca do sistema de saúde e as práticas discentes restritas a estabelecimentos públicos eram problemas que dificultavam a discussão ampla do sistema de saúde no País.
Em 1993, foi iniciada na FMRP-USP a implantação de uma Reforma Curricular ${ }^{1,2}$ que compactou os conteúdos teóricos ao eliminar repetições, ampliou os campos de estágios e os períodos de prática discente, e estimulou inovações didáticopedagógicas em praticamente todos os departamentos da Unidade $^{3}$. Os ciclos básico e pré-clínico foram limitados a dois anos cada um, liberando os dois últimos anos do curso para o internato - o primeiro totalmente desenvolvido na rede básica de serviços de saúde, e o outro no Hospital Universitário.

Quando as mudanças do currículo chegaram ao quarto ano, em 1996, a disciplina de OAS foi antecipada para o oitavo semestre e foi reduzida para 40 horas - uma semana em tempo integral - com três turmas por ano. Inicialmente, a disciplina passou a ser desenvolvida no seu formato tradicional com seminários e aulas teóricas, oferecendo apostilas sobre os temas principais e copiando e distribuindo material obtido do Ministério da Saúde, Secretaria de Estado da Saúde de São Paulo, Instituto Brasileiro de Geografia e Estatística (IBGE), etc.

Todavia, este curso "compacto" impunha uma rotina desgastante, desestimulando a participação e a criatividade de alunos e professores, forçando a edição do material docente para todos os alunos. Além disso, esse material, muitas vezes, precisava ser reeditado repetidamente porque se tornava defasado com rapidez - caracterizando uma situação semelhante à da crise da educação tradicional ${ }^{4}$-, estimulando os docentes a procurar modelos educacionais alternativos.

No ano 2000, a USP disponibilizou, no campus de Ribeirão Preto, um software para promover o ensino à distância, o aplicativo canadense Web Course Tools (WebCT) ${ }^{5}$ e, após um breve treinamento e um curso introdutório, decidimos implementar uma nova proposta pedagógica no curso.

\section{OBJETIVOS DO NOVO CURSO}

\section{Objetivos Educacionais Terminais}

Melhorar a pesquisa e aprendizagem acerca do sistema de assistência à saúde no Brasil. Conhecer a estrutura e organização dos serviços de saúde. Compreender os problemas do financiamento da assistência à saúde. Analisar as características do modelo médico-assistencial no Brasil. Dar noções da avaliação da assistência à saúde e aplicar indicadores. Avaliar criticamente sistemas municipais de saúde.

\section{Objetivos Educacionais Intermediários}

Aumentar o interesse e motivação dos alunos em relação aos conteúdos do curso. Aprender a acessar fontes oficiais de informações de saúde. Promover a aprendizagem cooperati- 
va. Desenvolver habilidades cognitivas, como organizar tarefas e processos. Aprender a transformar informações e não reproduzi-las, apenas. Incentivar a criatividade, utilizando tecnologias da informação e comunicação.

\section{EMBASAMENTO TEÓRICO DA NOVA PROPOSTA}

A nova proposta do curso de OAS-RCG 452 se aproxima das concepções da educação baseadas no construtivismo ${ }^{6,7}$, adotando a estratégia de provocar, desde o início, uma intensa motivação como base do processo de pesquisa-aprendizagem.

O escopo do curso é interpretar o sistema de saúde no Brasil, sistema com o qual o futuro profissional deverá interagir. Além disso, o curso lhe oferece a oportunidade de estudar os sistemas municipais de saúde, de forma a poder orientar a escolha futura do local de trabalho. Ambos os objetivos serão atingidos ao se procurar conhecer a realidade da saúde no Brasil - exercitando as relações teoria-prática e sujeito-objeto, com o auxílio de conteúdos teóricos discutidos e sintetizados ao longo de seminários presenciais obrigatórios.

Neles, a meta é adquirir o domínio de conceitos como necessidades e demanda, modelos de assistência, direito à saúde e financiamento de serviços, sistemas assistenciais, avaliação da assistência, ultrapassando o nível operativo dos mesmos para alcançar o nível mais abstrato da interpretação do sistema de saúde. Outro aspecto privilegiado no curso é o trabalho cooperativo que se desenvolverá nos grupos moderadores que fazem a síntese das discussões realizadas pela rede e, outra vez, na elaboração do relatório do trabalho final.

Com estes objetivos pedagógicos, encontramos no WebQuest ${ }^{8}$ - modelo de pesquisa e aprendizagem pela rede proposto por Bernie Dodge ${ }^{9}$ - o substrato teórico e operacional adequado. O WebQuest permite promover a aprendizagem cooperativa, a organização de tarefas, a elaboração de dados e incentivar a criatividade dos alunos. Desta forma, podemos propiciar o contato com fontes oficiais de dados e informações durante o processo de pesquisa e aprendizagem, permitindo modificar os objetivos educacionais tradicionais de responder perguntas e resolver problemas.

O novo curso proposto permitiu também definir objetivos educacionais em todos os níveis de complexidade definidos por Bloom ${ }^{10}$ : conhecimento, compreensão, aplicação, análise, síntese e avaliação.

\section{DINÂMICA DO CURSO}

Ao iniciarem o curso, os alunos recebem breve preleção acerca do modelo pedagógico adotado pela disciplina e sua relação com os objetivos educacionais terminais e intermedi- ários, enfatizando que a experiência poderá ser de grande valor em outros cursos da graduação e na futura vida profissional. Cada aluno recebe uma folha de iniciação com as instruções para fazer o cadastro no portal do curso, o nome de identificação e a senha correspondente, e breve descrição dos recursos disponíveis. Daí em diante, o aluno é estimulado a ler seguindo a ordem exposta no sumário: apresentação, programa do curso e todos os demais conteúdos, instruções e atividades obrigatórias.

O novo formato do curso, inteiramente estruturado com a utilização dos recursos do WebCT, reorganizou os conteúdos do programa em cinco eixos temáticos integradores, um para cada dia de aula: 1. População e Serviços de Saúde Indicadores da Assistência; 2. Estrutura e Organização dos Serviços de Saúde - Níveis assistenciais e Regionalização; 3. Financiamento e Custos da Assistência à Saúde; 4. Sistemas de Assistência à Saúde; 5. Avaliação da Assistência Médica e Direitos do Paciente.

Para cada eixo temático foi elaborado um roteiro de estudo e pesquisa que inclui, com o recurso de hipertextos, links para fontes de dados ou referências de documentos oficiais. A discussão temática é desenvolvida em dois tempos ou fases: a primeira é a discussão antecipada com a utilização dos recursos do fórum - em forma de "Webminar" ou seminário pela rede. Todos os alunos devem participar com ao menos uma mensagem com suas considerações acerca das questões propostas no roteiro ou outras, de própria lavra.

O professor acompanha as questões propostas no fórum e, sempre que necessário, intervém com observações e/ou esclarecimentos. As questões dos alunos são sistematizadas por um Grupo Moderador (seis ou sete alunos de uma classe de 33) e apresentadas e discutidas com o professor na segunda fase-no seminário presencial de síntese temática que ocorre diariamente. As instruções para orientar o trabalho dos Grupos Moderadores são publicadas em página especial com a escala de apresentação dos mesmos, renovada a cada turma.

Os alunos também devem realizar, individualmente ou em duplas, um trabalho prático, que consiste no estudo/pesquisa do sistema municipal de saúde de um município de livre escolha do aluno. É fornecido um roteiro-guia para a elaboração do trabalho, com uma lista dos itens que deverão ser considerados: população, atividades econômicas, características geográficas, perfis da morbidade e mortalidade, serviços de saúde existentes, financiamento da saúde, programas de saúde oferecidos, etc. O roteiro inclui os portais de pesquisa mais importantes, mas os alunos são estimulados a procurar outras fontes, a fim de enriquecer as referências disponibilizadas no curso. 
O sistema WebCT permite a inserção de "dicas" (tips) mensagens que se abrem aleatoriamente sempre que o aluno entra no Curso -, as quais reforçam as instruções, alertam sobre prazos, orientam condutas, etc. Foram elaboradas 14 dicas de reforço, que os alunos também podem acessar e ler voluntariamente, de forma corrida.

\section{USO DE TECNOLOGIAS E PERFIL DO ALUNO}

Nos cursos da área da saúde, a maior parte das disciplinas desenvolve seus conteúdos teóricos em aulas de formato tradicional e seminários; aulas seguidas tornam a freqüência aos cursos uma tarefa cansativa tanto para os alunos como para os professores.

Por outro lado, os novos alunos na área da saúde são muito familiarizados com o uso de recursos de informática; a grande maioria deles possui computador em casa e não tem dificuldades com o acesso e pesquisa na rede. No entanto, para maior facilidade do ensino e pesquisa discentes, a Faculdade de Medicina instalou uma sala com 30 computadores (Pro-Aluno), ligados em rede e com acesso à internet.

Os livros de textos nas áreas da saúde são geralmente caros, o que limita o número de livros que os alunos podem adquirir. Mas a rede provê enciclopédias médicas, textos de atualização e notícias sobre questões científico-tecnológicas e mudanças organizacionais no setor.

Desta forma, utilizar tecnologias para acessar conteúdos didáticos representa uma boa alternativa aos custos e às rotinas extenuantes, porque permite o acesso e a leitura de textos indicados-nacionais ou estrangeiros-sem maiores dificuldades. Além disso, cria amplas possibilidades de explorar novos materiais correlatos de interesse para o aprimoramento do processo de aprendizagem, respeitando os tempos e o perfil do aluno.

\section{USO DE TECNOLOGIAS E OBJETIVOS DO CURSO}

A Reforma Sanitária, que enfatizou o controle social, transformou a área da saúde num dos setores sociais com maior divulgação de dados, informações, documentos e relatórios, tornando árdua a tarefa de se manter informado acerca das mudanças nos cenários municipal, estadual e federal. Este imenso acervo de dados oficiais, disponibilizado on line, é fonte obrigatória de consulta para profissionais de serviços, gestores e educadores, e se transformou no mais completo campo de pesquisa e aprendizagem na área da saúde, como fonte de consulta, atualização, levantamento de informações, pesquisas bibliográficas, trabalhos comparativos, busca e intercâmbio de experiências. Neste contexto, é difícil, hoje, estudar o sistema de saúde no Brasil sem a utilização das tecnologias da informação e comunicação.

\section{SISTEMA DE AVALIAÇÃO}

Metade da nota final individual do curso de OAS provém da participação do aluno nas atividades de estudo e discussão de apostilas e documentos oficiais disponibilizados e dos comentários produzidos acerca dos cinco eixos temáticos da saúde no Brasil. Esta primeira parte da atividade é realizada por meio do fórum já citado, elaborando-se questões e comentando-se as mensagens dos outros alunos no desenvolvimento de um seminário de discussão pela rede - "Webminar". O estudo de cada eixo temático é concluído com uma reunião ou seminário presencial de síntese, que ocorre diariamente com a presença obrigatória de todos os alunos e a Coordenação do Grupo Moderador. O Grupo Moderador do dia (cinco Grupos Moderadores, com seis a sete alunos) se encarrega de apresentar uma sistematização da discussão no fórum, destacando os comentários, assuntos pendentes, dificuldades e sugestões.

Tanto no fórum como na reunião presencial, é importante demonstrar: entendimento da matéria, capacidade de comparar, elaborar informações, pesquisar questões subjacentes, descobrir novos elementos (inclusive portais de informações), capacidade de análise da situação de saúde e de conclusão de forma sintética.

A outra metade da nota provém do trabalho prático, pesquisa sobre o sistema de assistência à saúde num município de escolha do aluno, de preferência aquele onde reside ou onde imagina um dia trabalhar. O relatório do trabalho prático deve incluir dados quantitativos e qualitativos pesquisados, elaborados e analisados pelos alunos, com comentários próprios sobre a situação encontrada, incluindo as perspectivas para o exercício profissional naquela localidade.

Embora a plataforma utilizada (software) registre automaticamente a "navegação" dos alunos no curso, fornecendo estatísticas do número de entradas no curso, acessos a cada uma das páginas, número de mensagens lidas e de mensagens enviadas, consideramos estes dados de valor apenas indicativo, do ponto de vista quantitativo, da intensidade das atividades do aluno no curso ou de dificuldades de acesso ao mesmo, e não devemos dispensar a avaliação qualitativa da produção discente. Foi inserido um ícone de auto-avaliação (my progress) que permite ao aluno o acesso aos indicadores quantitativos de suas próprias atividades. Finalmente, os critérios de avaliação da participação e do trabalho são "publicados" no curso, em página específica.

Quanto à avaliação do desempenho dos alunos, os melhores indicadores são: a profundidade com que os temas são debatidos no seminário presencial de síntese final de cada eixo temático e a qualidade da elaboração e análise dos dados ob- 
tidos nas pesquisas dos sistemas municipais de saúde. Na última edição do curso, praticamente a metade da classe mereceu nota final igual ou maior que 8,0, fato muito raro no modelo tradicional. Mereceram destaque, pela análise e avaliação do sistema municipal, os relatórios dos municípios de Três Lagoas (MS), Ribeirão Preto, Piracicaba, Santos (SP) e Araxá (MG); pela criatividade na apresentação, os de Marília, Botucatu, Batatais, Franca e Santo André (SP).

Este formato educacional vem tendo aprovação crescente por parte dos alunos, estando acima de dois terços no curso de medicina, onde se iniciou. Nos cursos de Medicina, Fisioterapia, Terapia Ocupacional e Informática Biomédica, a aceitação é de cerca de $90 \%$, apurados em questionários individuais da disciplina aplicados ao final da mesma.

A aceitação dos alunos pode ser atribuída ao fato de o modelo proposto ser mais econômico: a leitura do texto e a visita a portais podem ser realizadas em tempo menor do que seria necessário numa aula teórica. E também ao fato de reconhecerem a importância da atividade prática realizada como um exercício que poderá ser de grande utilidade na futura vida profissional.

Para a equipe docente, o modelo é positivo porque evita a repetição mecânica das aulas e oferece a oportunidade de participar de discussões mais aprofundadas no seminário presencial de síntese final do que em seminários tradicionais. Como os alunos têm acesso a todo o material e liberdade de aprofundar o conhecimento sobre temas de seu interesse por meio de novas pesquisas, cada situação educacional é muito dinâmica e singular, exigindo dos docentes atualização constante de muitos pontos do curso, às vezes num mesmo semestre.

\section{AVALIAÇÃO DA EXPERIÊNCIA}

Considerando a pesquisa-aprendizagem no ensino de políticas e gestão de saúde, nosso principal elemento inovador é a elaboração de uma proposta educacional baseada na integração de conteúdos teóricos com a utilização da rede web como instrumento de pesquisa e a orientação e a execução de uma atividade prática voltada à interpretação e avaliação da assistência à saúde no Brasil.

Alguns pontos específicos destacam-se na avaliação: 1. a motivação dos alunos, pela estratégia de propor a pesquisa e avaliação do sistema de saúde de um município de livre escotha dos alunos, com uso dos recursos da rede; 2. a promoção de uma posição proativa dos alunos, por meio da elaboração de roteiros ou gabaritos para a realização de tarefas de estudos e pesquisa-aprendizagem em saúde; 3. a promoção da inclusão digital, pelo conhecimento e utilização de portais institucionais oficiais de interesse permanente nas questões de política e gestão de saúde; 4 . o desenvolvimento de um ambiente virtual de pesquisa-aprendizagem disponível 24 horas por dia que substitui com vantagens o tempo antes dedicado à presença obrigatória em sala de aula.

Outros aspectos positivos foram: a facilidade de replicação da disciplina em outros cursos da área da saúde e a aceitação pelos alunos do novo modelo pedagógico.

A presente experiência teve início na reformulação da disciplina de Organização e Administração em Saúde (RCG 452) do curso de graduação em Medicina (cem alunos, em três turmas) da Faculdade de Medicina de Ribeirão Preto da USP. O sucesso alcançado permitiu estender o modelo criado aos outros cursos da Faculdade de Medicina: Fisioterapia (40 alunos) e Terapia Ocupacional (20 alunos) em 2002; Fonoaudiologia (30 alunos), Nutrição e Metabolismo (30 aluno) e Informática Biomédica (40 alunos), de maneira que em 2003 houve oito edições, com um total de 260 alunos. Este modelo pedagógico foi expandido a partir de 2001 para o ensino de pósgraduação, na disciplina de Política de Saúde do mestrado em Saúde na Comunidade, com adesão ainda mais entusiasta dos alunos. Finalmente, tendo a USP suspendido o contrato com a WebCT Inc. em dezembro de 2004, a partir de 2005 utilizamos a plataforma para ensino a distância TelEduc ${ }^{11}$ - produto nacional, disponível gratuitamente, desenvolvida pelo Núcleo de Informática Aplicada à Educação e Instituto de Computação da Universidade de Campinas, SP - com maior aceitação e igual sucesso.

\section{REFERÊNCIAS}

1. Rodrigues MLV. Inovações no Ensino Médico e outras mudanças: aspectos históricos na Faculdade de Medicina de Ribeirão Preto-USP. Medicina (Ribeirão Preto). jul./set. 2002; 35: 231-235.

2. Troncon LEA et al. Avaliação de uma reestruturação curricular na Faculdade de Medicina de Ribeirão Preto . Rev Bras Educ Méd. 2004; 28(2): 145-155.

3. Yazlle JSR. Departamento de Medicina Social. A reestruturação e os novos tempos. Medicina (Ribeirão Preto). 2002; 35: 306-312.

4. Morán JM. Mudar a forma de ensinar e de aprender com tecnologias. Transformar as aulas em pesquisa e comunicação presencial-virtual. Disponível em: http:// www.eca.usp.br/prof/moran/uber.htm Acesso em: 28/ $02 / 2002$

5. Web Course Tools Inc. Disponível em: http:// www.webct.com/webct Acesso em: 21/05/2000 
6. Moura AMM, Azevedo AMP, Mehlecke Q. As Teorias de Aprendizagem e os Recursos da Internet Auxiliando o Professor na Construção do Conhecimento. Disponível em: http:/ / www.abed.org.br/publique/cgi/cgilua.exe/sys / start.htm? UserActiveTemplate $=4$ abed\&infoid $=188 \&$ sid $=102$

7. Franco SRK. O Construtivismo e a Educação. 9. ed. Porto Alegre: Mediação; 2004.

8. Escola do Futuro da USP/WebQuest. Disponível em: http:www.futuro.usp.br

9. Dodge B. FOCUS: Five Rules for Writing a Great WebQuest. Disponível em: http: / /www.futuro.usp.br/projetos / proj_webquest.htm

10. Taxonomia dos Objetivos educacionais de Blomm. Disponível em: http:/ / www.webquest.futuro.usp.br/recursos / bloom.html

11. TelEduc Disponível em: http://www.edumed.org.br/ teleduc. Acesso em 26/02/2005.

\section{ANEXO}

Principais Portais de Pesquisa Disponibilizados no Curso

- Agência Nac. de Saúde Suplementar: www.ans.gov.br /

- Agency Healthcare Research Quality: www.ahcpr.gov

- Ass. Bras. P. G. Saúde Coletiva: www.abrasco.org.br/

- Ass. Bras. Educação a Distância: www.abed.org.br/

- Ass. Médica Brasileira: www.amb.org.br/site/index.php3

- Associação Paulista Saúde Pública: www.apsp.org.br/

- Association Health Serv Research: www.ashr.org

- Biblioteca Rev. Cient. Saúde Pública: www.scielosp.org/

- Biblioteca Virtual Saúde Pública MS: www.saudepublica.bvs.br/

- Conferência Nac. Saúde On Line: www.datasus.gov.br/cns/datasus.htm

- Conselho Nacional de Saúde: www.conselho.saude.gov.br/

- Conass: www.conass.com.br/

- Conasems: www.conasems.com.br/

- C. Política RHMS: dtr2001.saude.gov.br/sps/depart/cgprh/estatisticas / emprego.html
- Datasus: www.datasus.gov.br

- Informações em Saúde: tabnet.datasus.gov.br/tabnet/tabnet.htm

- Faculdade de Saúde Pública / USP: www.fsp.usp.br /

- Fundação Oswaldo Cruz: www.fiocruz.br/

- Fundação Seade: www.seade.gov.br

- Hospital das Clínicas da FMRP / USP hcrp.fmrp.usp.br/

- IBGE: www.ibge.gov.br

- IBGE - Banco de Dados Agregados: www.sidra.ibge.gov.br/

- Ipea: www.ipea.gov.br/

- Medicamentos Essenciais, OMS: www.who.int / medicines / organization / dap / orgdapmain.shtml

- Medlineplus / Enciclopédia Médica: medlineplus.gov/spanish

- Ministério da Saúde: www.saude.gov.br

- Org. Pan. da Saúde, PAHO: www.paho.org

- Org. Mundial da Saúde, WHO: www.who.org

- Org. Mun. Saúde, Europe: www.who.dk

- Planos de Saúde - Custos: www.francosegurosaude.com.br/

- Portal de Buscas: www.google.com/

- Programa Saúde da Família - MS: dtr2001.saude.gov.br/psf//index.htm

- Revistas Científicas Saúde Pública: www.scielosp.org/

- Sec. Estadual de Saúde (SP): www.saude.sp.gov.br

- Universiabrasil: www.universiabrasil.net

- U. S. National Library Medicine: www.nlm.nih.gov/

\section{Endereço para correspondência}

Departamento de Medicina Social

Faculdade de Medicina de Ribeirão Preto - USP

Av. Bandeirantes, 3.900 - Campus da USP

14049-900 - Ribeirão Preto - SP

Juan Stuardo Yazlle Rocha

e-mail: jsyrocha@fmrp.usp.br 